\title{
Effect of cyproheptadine on weight gain in malnourished children: a randomized, controlled trial
}

\author{
Sanguansak Rerksuppaphola ${ }^{\mathrm{a}}$ Lakkana Rerksuppaphol $^{\mathrm{b}}$ \\ ${ }^{a}$ Department of Pediatrics, ${ }^{b}$ Department of Preventive Medicine, Faculty of Medicine, Srinakharinwirot \\ University, Nakhorn-Nayok 26120, Thailand
}

\begin{abstract}
Background: Cyproheptadine has been used therapeutically as an appetite stimulant in various chronic illnesses. However, no clinical data are available on the therapeutic effect of cyproheptadine in malnourished children without underlying pathological conditions.

Objective: Investigate the short-term effect of cyproheptadine on weight gain in malnourished children who appear otherwise normal on physical examination.

Methods: Seventy malnourished children who were otherwise normal on physical examination were recruited to participate in a randomized, double-blind, placebo-controlled trial. Thirty-seven children were randomized to a treatment regimen of cyproheptadine $(0.1 \mathrm{mg} / \mathrm{kg} / \mathrm{dose}$, three times/day for eight weeks), and 33 children were randomized to receive placebo over a period of eight weeks. Subjects were evaluated at a baseline visit and at four visits at two-week intervals. Parameters assessed included baseline demographics, anthropometrics (weight, height, skin-fold thickness, waist and hip circumferences, and fat composition by bioelectric impedance analysis), adverse events, and pill counts. Data were analyzed by Student's t-test and Chi-square test; a p- value $<0.05$ was considered significant.

Results: No significant differences were observed in baseline demographic characteristics and anthropometric parameters between the groups. The cyproheptadine-treated group showed a significantly greater weight gain over the baseline compared with the control group. The absolute weight gain was significantly higher in the cyproheptadine-treated group than in the control group at the end of study. No significant difference was observed in the change in the body fat percentage between the groups. No serious adverse events were reported. Adverse events included mild sedation, nausea, diarrhea, abdominal pain, and headache. No significant differences in the frequency of adverse events were observed between the groups.

Conclusions: Cyproheptadine treatment was well tolerated and resulted in significant weight gain in malnourished children, without increasing the body fat percentage.
\end{abstract}

Keywords: Child, cyproheptadine, malnutrition, randomized controlled trial, weight gain

Cyproheptadine is an antihistaminic and antiserotonergic agent. It has been approved by the Thai Food and Drug Administration for the treatment of allergic conditions [1]. A favorable side effect of cyproheptadine is its appetite-stimulating action, which has been exploited therapeutically in the treatment

Correspondence to: Sanguansak Rerksuppaphol, MD, Faculty of Medicine, Srinakharinwirot University, 62 M7 Rangsit-Onkhaluck Rd. Onkhaluck, Nakhorn-Nayok 26120, Thailand.E-mail: sanguansak_r@hotmail.com of chronic illnesses such as cystic fibrosis [2], malignancies [3-6], anorexia nervosa [7], AIDS [8], and renal failure [9]. However, cyproheptadine has not been used clinically in malnourished children who are otherwise normal. Poor appetite leading to poor weight gain is a characteristic finding among underweight children without underlying pathological conditions.

In this study, we investigated the role of cyproheptadine as an appetite stimulant by using weight gain as the end-point and determined its efficacy and tolerability in malnourished children. 


\section{Materials and methods}

The effect of cyproheptadine on weight gain in malnourished children was studied in a randomized, double-blind, placebo-controlled trial conducted by the Pediatric Nutrition Clinic, Srinakharinwirot University Hospital, between June and December 2008. Seventy children (age: 6-15 years), who had recently been diagnosed as underweight and who appeared normal on physical examination, were recruited for the study. A body weight of less than $90 \%$ of the predicted body weight for a given age and gender was defined as underweight for purposes of this study. Children with chronic illnesses, congenital abnormalities, or allergy to antihistaminic agents were excluded from the study.

The study protocol was approved by the Ethics Committee of Faculty of Medicine, Srinakharinwirot University. Written informed consent was obtained from the parents or legal guardians of all study participants.

The study participants were randomized using a computer program: 37 children received cyproheptadine $(0.1 \mathrm{mg} / \mathrm{kg} /$ dose, three times/day) and 33 children received a placebo over a period of eight weeks. Demographic profiles and baseline anthropometric data, including weight, height, skin-fold thickness, mid-upper arm circumference (MUAC), waist and hip circumferences, and fat composition (measured by bioelectric impedance analysis), were obtained. Measurements of weight, height, and MUAC were done in accordance with the WHO Expert Committee guidelines [10]. Weight was measured to the nearest $100 \mathrm{~g}$, and height, to the nearest millimeter. Body mass index (BMI) was calculated as weight $/(\text { height })^{2}\left[\mathrm{~kg} / \mathrm{m}^{2}\right]$. MUAC was measured midway between the tip of the left shoulder blade and the tip of the elbow, with a normal non-stretch tape to the nearest $0.1 \mathrm{~cm}$. Waist and hip circumferences were measured in the upright position at the narrowest girth in the waist area and at the level of the greatest posterior protuberance of the buttocks, respectively. Triceps skin fold (TSF), biceps skin fold (BSF), sub-scapular skin fold (SSSF), and supra-iliac skin fold (SISF) were measured on the left side, using a Lange skin-fold caliper. The children returned for four follow-up visits at two-week intervals and were weighed by a registered nurse at each visit on the same scales. Adverse events and compliance with treatment were monitored at each visit based on parent reports and pill counts, respectively.

Weight, skin-fold thickness, MUAC, waist and hip circumferences, and body fat composition were measured at the end of the study.

\section{Statistical analysis}

The results were presented as mean, standard deviation (SD), and percent values. Pearson chi-square was used to compare proportions between the two groups. Student's t-test was used to compare the two groups at the baseline visit and at each follow-up visit thereafter. The paired t-test was used within each group to compare various parameters at each visit with the values at the baseline visit. A p-value $<0.05$ was considered statistically significant. All analyses were carried out using the SPSS 11.0 software package.

\section{Results}

Seventy underweight children (mean age: 11.3 years) completed the study. The study group comprised $61.4 \%$ boys. The average baseline weight, height, BMI, and body fat composition of the cyproheptadinetreated group $\left(25.5 \mathrm{~kg}, 132.2 \mathrm{~cm}, 14.8 \mathrm{~kg} / \mathrm{m}^{2}\right.$, and $9.6 \%$, respectively) were similar to those of the control group $\left(25.3 \mathrm{~kg}, 129.5 \mathrm{~cm}, 14.8 \mathrm{~kg} / \mathrm{m}^{2}\right.$, and $9.6 \%$, respectively). The baseline demographic and anthropometric data of the children in both groups are shown in Table 1. The mean body weights of the cyproheptadine-treated group and the control group were 25.8 and $25.7 \mathrm{~kg}$ at two weeks, 26.0 and $25.4 \mathrm{~kg}$ at four weeks, 25.9 and $25.9 \mathrm{~kg}$ at six weeks, and 26.8 and $25.9 \mathrm{~kg}$ at eight weeks, respectively. Significant weight gain occurred in both groups as early as week 2 of treatment $(\mathrm{p}<0.05)$ compared with the baseline body weight. Children in the cyproheptadinetreated group showed a greater increase in weight over the baseline weight at every follow-up visit than the children in the control group (Table 1).

The change in body fat from the baseline visit until the end of the study did not significantly differ between the two groups (cyproheptadine $1.01 \%$ vs. control $0.31 \% ; p=0.54$ ). The change in skin fold thickness, and hip and waist circumferences from the baseline visit until the end of the study also did not significantly differ between the two groups (Table 2). 
Table 1. Comparison of baseline parameters. Data are represented in mean (SD).

\begin{tabular}{llll}
\hline & Placebo & Cyproheptadine & P-value \\
\hline Sex $(\mathrm{M} / \mathrm{F})$ & $23 / 10$ & $20 / 17$ & 0.22 \\
Age $($ year) & $11.37(2.00)$ & $11.15(2.31)$ & 0.69 \\
Weight $(\mathrm{kg})$ & $25.26(6.53)$ & $25.52(6.71)$ & 0.87 \\
Height $(\mathrm{cm})$ & $129.50(12.96)$ & $130.22(12.60)$ & 0.82 \\
BMI $\left(\mathrm{kg} / \mathrm{m}^{2}\right)$ & $14.80(1.15)$ & $14.79(1.61)$ & 0.95 \\
TSF $(\mathrm{mm})$ & $6.18(1.70)$ & $5.81(1.65)$ & 0.36 \\
BSF $(\mathrm{mm})$ & $4.52(2.11)$ & $4.35(1.80)$ & 0.73 \\
SSSF $(\mathrm{mm})$ & $5.15(1.39)$ & $5.22(1.27)$ & 0.84 \\
SISF (mm) & $4.85(2.39)$ & $4.95(3.18)$ & 0.89 \\
MUAC $(\mathrm{cm})$ & $14.94(2.98)$ & $15.08(2.93)$ & 0.84 \\
Waist circumference (cm) & $51.91(6.30)$ & $52.02(6.49)$ & 0.92 \\
Hip circumference (cm) & $63.70(7.16)$ & $64.96(9.03)$ & 0.53 \\
Waist/hip ratio & $0.80(0.49)$ & $0.79(0.05)$ & 0.20 \\
Body fat composition $(\%)$ & $9.62(4.14)$ & $9.58(5.32)$ & 0.98
\end{tabular}

BMI=body mass index, TSF=triceps skin fold, BSF=biceps skin fold, SSSF=sub-scapular skin fold, SISF=supra-iliac skin fold, MUAC=mid-upper arm circumference.

Table 2. Anthropometric parameters on follow-up visits. Data are represented in mean (SD).

\begin{tabular}{llll}
\hline & Placebo & Cyproheptadine & P-value \\
\hline $\begin{array}{l}\text { Weight gain from the baseline (kg) } \\
\quad\end{array} \quad$ t 2 weeks & $0.14(0.38)$ & $0.89(0.66)$ & $<0.01$ \\
$\quad$ at 4 weeks & $0.27(0.47)$ & $1.17(0.85)$ & $<0.01$ \\
$\quad$ at 6 weeks & $0.50(0.44)$ & $1.22(0.89)$ & $<0.01$ \\
$\quad$ at 8 weeks & $0.63(0.66)$ & $1.25(0.81)$ & $<0.01$ \\
TSF (mm) & $6.45(1.70)$ & $7.11(1.90)$ & 0.14 \\
BSF(mm) & $4.85(1.77)$ & $5.32(2.30)$ & 0.34 \\
SSSF (mm) & $5.30(1.19)$ & $5.86(1.34)$ & 0.07 \\
SISF (mm) & $7.09(2.02)$ & $8.27(2.84)$ & 0.05 \\
MUAC (cm) & $14.32(1.71)$ & $14.89(3.44)$ & 0.39 \\
Waist circumference (cm) & $50.91(4.21)$ & $50.92(5.30)$ & 0.99 \\
Hip circumference (cm) & $63.52(6.19)$ & $64.78(6.74)$ & 0.42 \\
Body fat composition (\%) & $9.93(3.68)$ & $10.59(4.51)$ & 0.51 \\
\hline
\end{tabular}

TSF=triceps skin fold, BSF=biceps skin fold, SSSF=sub-scapular skin fold, SISF=supra-iliac skin fold, MUAC=mid-upper arm circumference.

\section{Adverse events}

No severe adverse events resulted in treatment withdrawal in either of the groups. The adverse events recorded in the cyproheptadine-treated group were mild sedation (24.3\%), nausea (5.4\%), abdominal pain (5.4\%), diarrhea (5.4\%), and headache (2.7\%), whereas in the control group, mild sedation (12.1\%), nausea (3.0\%), abdominal pain (6.1\%), and headache (3.0\%) were reported. No statistically significant differences were present between the two groups for any of the adverse events.

\section{Treatment compliance}

Treatment compliance was calculated as the percentage of total intake doses from the total eligible dose and was high in both groups $(82.3 \%$ in the cyproheptadine-treated group and $81.8 \%$ in the control group, $\mathrm{p}=0.80$ ). 


\section{Discussion}

The present results show that cyproheptadine at a daily dose of $0.3 \mathrm{mg} / \mathrm{kg}$ resulted in weight gain among underweight children who were otherwise normal on physical examination. This effect was sustained from the second week of treatment through the end of study. Children in the cyproheptadinetreated group exhibited significantly higher weight gain than that exhibited by the children in the control group, with no significant differences in body fat indices between the groups.

The clinical significance of cyproheptadine administration to malnourished children has not been extensively studied [11]. Studies have documented the role of cyproheptadine therapy in promoting weight gain in children with chronic pathological conditions [2, 12, 13]. However, not much is known about the efficacy of cyproheptadine in malnourished children without underlying pathological conditions. Our results agree with the study by Mahachoklertwattana et al. [11]. They reported that cyproheptadine therapy resulted in an absolute weight gain of $0.66 \mathrm{~kg}$ in underweight children aged 2-10 years $(n=11)$ within two months, and the weight gain velocity effected by cyproheptadine therapy in undernourished children declines proportionately with time of treatment. In our study, we obtained a relatively early therapeutic response, starting in the second week of treatment. We used a higher dose of cyproheptadine $(0.3 \mathrm{mg} / \mathrm{kg}$ daily) than that $(0.1 \mathrm{mg} / \mathrm{kg}$ daily) used in a previous study [11]. Our findings suggest that the greater weight gain in our study is due to the dose-dependent effect of cyproheptadine on weight gain.

Body composition is roughly divided into two compartments, body fat and fat-free mass. Although studies have investigated the overall weight gain achieved by cyproheptadine therapy, no information is available on post-treatment body composition. Disproportionate weight gain in fat and fat-free mass could result in altered body composition and adiposity. Skin-folds, bioelectrical impedance analysis, and anthropometric studies are reliable and widely used in field and clinical settings to assess body composition [14,15]. Comparing baseline anthropometric indices to indicate body fat mass of the malnourished children in our study, we found that these indices were lower than reference standards of Asian children [15,16]. At the end of the study, we observed a significantly higher weight gain in the treatment group than in the control group, without significant difference in body fat percentage or other anthropometric indices indicating fat mass. This was an indirect indication that cyproheptadine treatment resulted in weight gain without changing the body fat composition. Height velocity was not measured in this study because of the relatively short follow-up period.

The mechanisms underlying cyproheptadinemediated weight gain are not well understood. Two hypotheses have been postulated to explain this phenomenon. The first hypothesis is based on the effect of cyproheptadine on the hypothalamic "feeding" center via its antihistamine and antiserotonin action[17-19]. Cyproheptadine was reported to stimulate appetite and caloric intake in animal [17, 20, 21] and human subjects [22-24]. The unreliability of recording food intake in children made it difficult to directly measure this appetite-stimulating effect [25]. However, most subjects reported an increased appetite at the end of the study when compared with the beginning of the study. The second hypothesis is based on the effect of cyproheptadine on the growth hormone and insulin-like growth factor (GH-IGF) system. Cyproheptadine therapy has been reported to increase the serum levels of insulin-like growth factorI (IGF-I) in addition to enhancing growth velocity in underweight children [11]. IGF-1, a GH-mediated growth factor, is now recognized as a potent mitogenic agent [26] and is one of the best indicators of growth hormone levels [27, 28]. Even though most of our subjects reported an increased appetite, an investigation of the exact mechanism of cyproheptadine action is beyond the scope of this study.

Compliance with cyproheptadine therapy in our study was $82 \%$, and the therapy was generally well tolerated. All adverse events reported on cyproheptadine therapy were consistent with the labeling on the package insert. Moreover, the drug related adverse events were mild and did not lead to discontinuation of the drug. The most commonly reported adverse event was mild sedation, a finding that is consistent with that of an earlier study [11].

In conclusion, we achieved significant weight gain without increase in body fat percentage among malnourished children on an 8-week regimen of cyproheptadine. Cyproheptadine was well tolerated. It is a good candidate for routine management of childhood malnutrition after larger sample sizes have been studied. 


\section{Acknowledgments}

We thank Mr. Adisorn Saengzue and Mrs. Kulvadee Robloo for their assistance in the study. The present study was supported by grants from Faculty of Medicine, Srinakharinwirot University. We have no conflict of interest to report.

\section{References}

1. Food and Drug Administration of Thailand. National list of essential medicine. [cited Dec 12 2009]. Available from: http://www.thaifda.com/ed2547/.

2. Homnick DN, Marks JH, Hare KL, Bonnema SK. Long-term trial of cyproheptadine as an appetite stimulant in cystic fibrosis. Pediatr Pulmonol. 2005; 40: 251-6.

3. Bruera E. Current pharmacological management of anorexia in cancer patients. Oncology (Williston Park). 1992; 6:125-30; discussion: 32, 37.

4. Jatoi A, Loprinzi CL. An update: cancer-associated anorexia as a treatment target. Curr Opin Clin Nutr Metab Care. 2001; 4:179-82.

5. Mattox TW. Treatment of unintentional weight loss in patients with cancer. Nutr Clin Pract. 2005; 20: 400-10.

6. Mao X, Liang SB, Hurren R, Gronda M, Chow S, $\mathrm{Xu}$ GW, et al. Cyproheptadine displays preclinical activity in myeloma and leukemia. Blood. 2008; 112: 760-9.

7. Powers PS, Santana C. Available pharmacological treatments for anorexia nervosa. Expert Opin Pharmacother. 2004; 5:2287-92.

8. Balog DL, Epstein ME, Amodio-Groton MI. HIV wasting syndrome: treatment update. Ann Pharmacother. 1998; 32:446-58.

9. Aguilera A, Selgas R, Diez JJ, Bajo MA, Codoceo R, Alvarez V. Anorexia in end-stage renal disease: pathophysiology and treatment. Expert Opin Pharmacother. 2001; 2:1825-38.

10. World Health Organization. Physical status: the use and interpretation of anthropometry. Report of a WHO expert committee. Technical Report Series No. 854. Geneva:WHO, 1995.

11. Mahachoklertwattana $\mathrm{P}$, Wanasuwankul S, Poomthavorn P, Choubtum L, Sriphrapradang A. Shortterm cyproheptadine therapy in underweight children: effects on growth and serum insulin-like growth factorI. J Pediatr Endocrinol Metab. 2009; 22:425-32.

12. Kaplowitz PB, Jennings S. Enhancement of linear growth and weight gain by cyproheptadine in children with hypopituitarism receiving growth hormone therapy. J Pediatr. 1987; 110:140-3.

13. Couluris M, Mayer JL, Freyer DR, Sandler E, Xu P, Krischer JP. The effect of cyproheptadine hydrochloride (periactin) and megestrol acetate (megace) on weight in children with cancer/treatmentrelated cachexia. J Pediatr Hematol Oncol. 2008; 30: 791-7.

14. Heyward VH. Practical body composition assessment for children, adults, and older adults. Int J Sport Nutr. 1998; 8:285-307.

15. Sung RY, So HK, Choi KC, Li AM, Yin J, Nelson EA. Body fat measured by bioelectrical impedance in Hong Kong Chinese children. Hong Kong Med J. 2009; 15:110-7.

16. Gharib NM, Shah P. Anthropometry and body composition of school children in Bahrain. Ann Saudi Med. 2009; 29:258-69.

17. Chakrabarty AS, Pillai RV, Anand BK, Singh B. Effect of cyproheptadine on the electrical activity of the hypothalamic feeding centres. Brain Res. 1967; 6: 561-9.

18. Montgomery AM, Fletcher PJ, Burton MJ. Behavioural and pharmacological investigations of 5-HT hypophagia and hyperdipsia. Pharmacol Biochem Behav. 1986; 25:23-8.

19. Sakata T, Ookuma K, Fukagawa K, Fujimoto K, Yoshimatsu H, Shiraishi T, et al. Blockade of the histamine H1-receptor in the rat ventromedial hypothalamus and feeding elicitation. Brain Res. 1988; 441:403-7.

20. Baxter MG, Miller AA, Soroko FE. The effect of cyproheptadine on food consumption in the fasted rat. Br J Pharmacol. 1970; 39:229P-30P.

21. Ghosh MN, Parvathy S. The effect of cyproheptadine on water and food intake and on body weight in the fasted adult and weanling rats. Br J Pharmacol. 1973; 48:328P-9P.

22. Rahman KM. Appetite stimulation and weight gain with cyproheptadine (periactin) in tuberculosis patients (double-blind clinical study). Med J Malaysia. 1975; 29:270-4.

23. Silverstone T, Schuyler D. The effect of cyproheptadine on hunger, calorie intake and body weight in man. Psychopharmacologia. 1975; 40:335-40.

24. Toth K, Szonyi A. The appetite stimulating and weight gain promoting effect of peritol (cyproheptadine) examined on a great number of outpatients. Ther Hung. 1976; 24:24-32.

25. Ambrosini GL, de Klerk NH, O’Sullivan TA, Beilin LJ, Oddy WH. The reliability of a food frequency 
questionnaire for use among adolescents. Eur J Clin Nutr. 2009; 63:1251-9.

26. Collett-Solberg PF, Misra M. The role of recombinant human insulin-like growth factor-I in treating children with short stature. J Clin Endocrinol Metab. 2008; 93: 10-8.

27. Lemaire P, Brauner N, Hammer P, Trivin C, Souberbielle JC, Brauner R. Improved screening for growth hormone deficiency using logical analysis data. Med Sci Monit. 2009; 15:MT5-10.

28. Spiliotis BE, Alexandrides TK, Karystianos C, Vassilakos P, Zadik Z, Nikolakopoulou NM, et al. The insulin-like growth factor-I (IGF-I) generation test as an indicator of growth hormone status. Hormones (Athens). 2009; 8:117-28. 